\title{
On the Features of Magnetogradient Planetary Waves in the Approximation of the Spherical Symmetry of the Ionosphere
}

\author{
G.V. Jandieri ${ }^{*}$, , A. Ishimaru ${ }^{2}$, V.G. Gavrilenko ${ }^{3}$, A.A. Surmava ${ }^{4}$ and A.I. Gvelesiani ${ }^{4}$ \\ ${ }^{I}$ Georgian Technical University, Institute of Cybernetics, Kostava street, 77, 0175, Tbilisi, Georgia \\ ${ }^{2}$ Department of Electrical Engineering, University of Washington, FT-10 Seattle, Washington 98195, USA \\ ${ }^{3}$ Nizhni Novgorod State University, Gagarin Avenue, 23, Nizhni Novgorod, 603950, Russia \\ ${ }^{4}$ M. Nodia Institute of Geophysics, 1 Alexidze Str, Tbilisi 0193, Georgia
}

\begin{abstract}
A problem of propagation of very long planetary waves in the upper atmosphere of the Earth is investigated. A new exact solution of the magnetohydrodynamics equations of the ionosphere in the spherical coordinate system is found taking into account the Earth's rotation and geomagnetic field. The general dispersion equation for the planetary waves in the $\mathrm{E}$ and $\mathrm{F}$ regions of the ionosphere is derived and propagation of these waves in weakly ionized ionospheric plasma are studied.
\end{abstract}

Keywords: Rotating ionosphere, electromagnetic planetary waves, Hall effect, dispersion equation.

\section{INTRODUCTION}

It is well known $[1,2]$ that only the internal waves are generated in the troposphere under the influence of the Coriolis force in the absence of dissipation effects, compressibility and temperature stratification (gyroscopic force gives additional stratification to the atmosphere and specific internal waves generate in it). They are called inertial waves in a short-wave approximation $\left(\lambda \leq 10^{3} \mathrm{~km}\right)$ and planetary Rossby waves in a long-wave approximation $\left(10^{3}-10^{4} \mathrm{~km}\right)$. The fundamental parameters of the Earth, such as the Earth's rotation frequency $\omega_{0}(\theta)$ and its latitudinal gradient $\nabla \omega_{0}$ are important in the dispersion equation of these waves. Wave motions disappear in the absence of rotation and the nonlinear Euler equation for the current function describes only convective motions of the troposphere.

Along with the parameters $\omega_{0}$ and $\nabla \omega_{0}$ also other fundamental parameters of the Earth, such as geomagnetic field $H_{0}(r, \theta)$ and its gradients become important in the ionosphere - (magnetic pressure $H_{0}^{2} / 8 \pi$ predominates over the pressures of neutrals and ionospheric plasma from 130 $\mathrm{km}$ and higher. Therefore electrodynamic effects dominate in the dynamic processes [3-5]).

In this paper a new exact solution of magnetohydrodynamics (MHD) equations considering the Earth's rotation and geomagnetic field is sought in the spherical coordinate system. It is shown that for the

*Address correspondence to this author at the Georgian Technical University, Institute of Cybernetics, Kostava street, 77, 0175, Tbilisi, Georgia;

Tel: +99532-227-406; Fax: +99532-253-898;

E-mails: jandieri@access.sanet.ge, jandieri_george@yahoo.com planetary scale wave processes, neglecting dissipation, compressibility and temperature stratification, the MHD equations of the ionosphere besides the well known hydrodynamic planetary Rossby waves contain both the slow $\left(5-100 \mathrm{~m} \mathrm{~s}^{-1}\right)$ ultra-low frequency $\left(10^{-5}-10^{-6} \mathrm{~s}^{-1}\right)$ planetary waves of hydrodynamic nature and ultrasonic (above $\left.1 \mathrm{~km} \mathrm{~s}^{-1}\right)$ low-frequency $\left(3-10^{-4} \mathrm{~s}^{-1}\right)$ planetary waves of electromagnetic nature.

Generation of slow waves is connected with electrostatic dynamo field (wind mechanism), and generation of fast waves - with vortex electrical field and electromagnetic Ampere force. The fast electromagnetic planetary waves in the local Cartesian coordinate system ( $\beta$ - plane approximation) were first theoretically discovered in $[6,7]$ and slow planetary waves in [6-8]. The latitudinal gradients of geomagnetic field play important role in dispersion equation of these waves. Therefore, following the terminology of Chernogor [9] we'll term this new oscillating branch of the ionospheric resonator as magnetogradient planetary waves. The influence of curvature of the lines of force of the geomagnetic field, Rayleigh and ionic frictions on the magnetogradient planetary waves were investigated in $[7,10]$. Linear and nonlinear problems of propagation of these waves in the dissipative E-region of the ionosphere were solved in [11-14]. Below we will not take into account the effects of viscosity, compressibility and temperature stratification $[3,15,16]$. Experimental verifications of existence of the magnetogradient planetary waves at the ionospheric levels are given in [7,9,17-19].

Numerous experimental observations show $[3,9,19]$ that in case of influence on the ionosphere from below (during the earthquakes, man-made explosions, launching of spacecrafts) and from above (during the magnetic storms, substorms, etc) planetary waves are often registered in the 
ionosphere. Wavelength of these waves equals or exceeds the Earth radius. Sphericity approximation of the Earth ( $\beta$ approximation) is not correct for such very long planetary waves and requires more exact calculation of the Earth's curvature. Below the problem of propagation of the very long magnetogradient planetary waves in the upper atmosphere including $E(80 \div 150 \mathrm{~km})$ and $F(150 \div 600 \mathrm{~km})$ regions of the ionosphere is investigated in the spherical coordinate system.

\section{FORMULATION OF THE PROBLEM AND THE INITIAL DYNAMIC EQUATIONS}

For the planetary scale waves instead of the Euler equation it is necessary to use Friedman's equation for the vorticity which naturally contains latitudinal gradients of the angular velocity of the Earth's rotation $\omega_{0}$ and geomagnetic field $\mathbf{H}_{0}$. The perturbed values of velocity $\boldsymbol{v}$ and induced magnetic field $\mathbf{h}$ together with the Maxwell's induction equation form the closed system $[3,7]$ :

$\operatorname{helm}\left(\operatorname{rot} \boldsymbol{v}+2 \boldsymbol{\omega}_{0}\right)=\Gamma, h e l m\left(\frac{\mathbf{h}}{\alpha \rho}+\frac{\mathbf{H}_{0}}{\alpha \rho}\right)=-\delta \Gamma$,

$\operatorname{div} \mathbf{v}=0, \operatorname{div} \mathbf{h}=0$,

where $\Gamma=\operatorname{rot} \mathbf{F}_{a}, \quad \mathbf{F}_{a}=\left[\operatorname{rot} \mathbf{h} \mathbf{H}_{0}\right] / 4 \pi \rho$ - Ampere force, $\alpha=c / e N$ - Hall parameter, $\rho=M N_{n}$ - density of the neutral particles, $N$ and $N_{n}$ - concentration of the ionospheric plasma and neutral particles, respectively; nondimensional parameter $\delta$ linking $E$ and $F$ layers is introduced for convenience: in the $E$ region of the ionosphere, where the Hall effect plays important role it equals to the unit $(\delta=1)$, in the $F$ region where the Hall effect is absent, $\delta$ is reduced to zero.

The vectors $\boldsymbol{\omega}_{0}$ and $\mathbf{H}_{0}$ in the spherical coordinate system have the components:

$\omega_{0}=\omega_{0 r}(\theta) \mathbf{e}_{r}+\omega_{0 \theta}(\theta) \mathbf{e}_{\theta}, \omega_{0 r}=\omega_{0} \cos \theta$,

$\omega_{0 \theta}=\omega_{0} \sin \theta, \mathbf{H}_{0}=H_{0 r}\left(r, \theta^{\prime}\right) \mathbf{e}_{r}+H_{0 \theta}\left(r, \theta^{\prime}\right) \mathbf{e}_{\theta}$,

$H_{0 r}=-2 H_{E} \cos \theta^{\prime}, H_{0 \theta}=-H_{E} \sin \theta^{\prime}, \omega_{0}=7,3 \cdot 10^{-5} \mathrm{~s}^{-1}$, $H_{E}=Q / r^{3}=0,31$ Gauss - value of the geomagnetic field at the equator, $H_{p}=2 H_{E}$ - value of the geomagnetic field at the pole, $Q=8,1 \cdot 10^{25}$ Gauss $\cdot \mathrm{cm}^{-3}$ - dipole magnetic moment of the geomagnetic field (further it is assumed that the geographical $r, \theta, \lambda$ and geomagnetic $r^{\prime}, \theta^{\prime}, \lambda^{\prime}$ coordinates coincide, i.e., it is considered that the magnetic axis is combined with the axis of the Earth's rotation), $r$ distance from the center of the Earth to the observation point, $\theta=90^{\circ}-\varphi$ - colatitude, $\varphi, \lambda$ - longitude counting to the east.

The operator helm, introduced by Friedman in honor of Helmholtz, for any vector field has the following form: $h e l m \mathbf{a}=\frac{\partial \mathbf{a}}{\partial t}-\operatorname{rot}[\mathbf{v} \mathbf{a}]+\boldsymbol{v} \operatorname{div} \mathbf{a}$

Friedman showed that the equality helm $\mathbf{a}$ to zero indicates conservation (freezing-in) of both the vector lines $\mathbf{a}$ and the intensity of its vector tubes helm $\mathbf{a}=0$, i.e., the vector $\mathbf{a}$ is invariant vector [3]. Invariant vector $\operatorname{rot} \boldsymbol{v}+2 \omega_{0}+e N \mathbf{H}_{0} / N_{n} M c$ for the $E$-region of ionosphere was first found in [3]. In the system (1.1) the vector $2 \omega_{0}$ is termed planetary vortex of the atmosphere [1]. Atmospheric particles in this vortex (as a solid body) rotate counterclockwise around the Earth (from the west to the east). Therefore vector $2 \omega_{0}$ is positive and directed from the south to the north. Let's term the vector $2 \boldsymbol{\Omega}_{i}=\mathbf{H}_{0} / \alpha \rho=e N \mathbf{H}_{0} / N_{n} M c=\mu \boldsymbol{\omega}_{i} \quad$ "magnetic" planetary vortex, $\mu=N / N_{n}$ characterizes a degree of ionization of the upper atmosphere, $\omega_{i}=e H_{0} / M c$ cyclotron frequency of the ions (as far as $e>0$ and $H_{0}<0$, we have $\left.\omega_{i}<0\right)$. In this vortex the particles of the ionic component of the upper atmosphere rotate clockwise in the magnetic field $\mathbf{H}_{0}$ (from the east to the west). Therefore vector $2 \boldsymbol{\Omega}_{i}$ is negative $\left(2 \boldsymbol{\Omega}_{i}<0\right)$ and is directed from the north to the south. From the expressions of the Ampere force $\left(\mathbf{F}_{a}=\frac{1}{4 \pi \rho}\left[\operatorname{rot} \mathbf{h} \mathbf{H}_{0}\right]=\left[\operatorname{rot} \frac{R^{2} \mathbf{h}}{\alpha \rho} \frac{c}{4 \pi e N} \frac{\mathbf{H}_{0}}{R^{2}}\right]\right) \quad$ follows existence of "magnetic" planetary vortex for the electrons $2 \boldsymbol{\Omega}_{e}=c \mathbf{H}_{0} / 4 \pi e N R^{2}=\left(c / c_{e}\right)^{2} \boldsymbol{\omega}_{e}, \quad$ where $c_{e}=R \omega_{e p}$, $\omega_{e p}=\sqrt{4 \pi e^{2} N / m}$ - is the plasma frequency of electrons, $\boldsymbol{\omega}_{e}=e \mathbf{H}_{0} / m c$ - cyclotron frequency of the electrons (as far as $e<0$ and $H_{0}<0$, the vortex $2 \boldsymbol{\Omega}_{\mathbf{e}}$ as $2 \boldsymbol{\omega}_{\mathbf{0}}$ is positive and is directed from the south to the north).

Introducing a vector potential of the magnetic field $\frac{\mathbf{H}}{\alpha \rho}=\frac{\mathbf{H}_{0}}{\alpha \rho}+\frac{\mathbf{h}}{\alpha \rho}=\operatorname{rot} \mathbf{U}_{0}+\operatorname{rot} \mathbf{U}$ from the system (1.1) we obtain:

$$
\begin{aligned}
& \frac{\partial \operatorname{rot} \mathbf{v}}{\partial t}-\operatorname{rot}[\mathbf{v} \operatorname{rot} \mathbf{v}]=\operatorname{rot}\left[\begin{array}{ll}
\boldsymbol{v} & 2 \omega_{0}
\end{array}\right]+\operatorname{rot} \mathbf{F}_{a}, \\
& \frac{\partial \operatorname{rot} \mathbf{U}}{\partial t}-\operatorname{rot}[\boldsymbol{v} \operatorname{rot} \mathbf{U}]=\operatorname{rot}\left[\begin{array}{ll}
\boldsymbol{v} & \left.2 \mathbf{\Omega}_{i}\right]-\delta \operatorname{rot} \mathbf{F}_{a},
\end{array},\right.
\end{aligned}
$$

$\operatorname{div} \mathbf{v}=0, \operatorname{div} \mathbf{U}=0$.

Since $\mathbf{U}$ has a dimension of velocity $\mathrm{m} \cdot \mathrm{s}^{-1}$, and "magnetic" planetary vortex $2 \boldsymbol{\Omega}_{i}$ dimension $\mathrm{s}^{-1}$, equations (1.2) and (1.3) formally describe the interaction of two incompressible fluids under the influence of gyroscopic forces, caused by the Earth's rotation, geomagnetic field $\left(\mathbf{F}_{k}=\rho\left[\begin{array}{ll}\boldsymbol{v} & 2 \boldsymbol{\omega}_{\mathbf{0}}\end{array}\right], \mathbf{F}_{H}=\rho\left[\begin{array}{ll}\boldsymbol{v} & 2 \boldsymbol{\Omega}_{\mathrm{i}}\end{array}\right]\right)$ and non-conservative Ampere force $\mathbf{F}_{a}$. 
In the absence of magnetic field $(\mathbf{H}=0)$, taking into account solenoidal nature of the vector $\boldsymbol{v}$, the equation (1.2) has the known exact solution:

$V_{r}^{\prime}=0, V_{\theta}=V_{\theta}^{\prime}(\theta, \lambda, t), \quad V_{\lambda}=\bar{V}_{\lambda}(\theta)+V^{\prime}(\theta, \lambda, t)$

$\bar{V}(\theta)=\alpha R \sin \theta$.

Here $V_{r}^{\prime}, V_{\theta}^{\prime}, \quad V_{\lambda}^{\prime}$ are the perturbations of velocity in the vertical, meridional and zonal directions. It is supposed that $V_{\theta}^{\prime}>0$ is directed to the south (in the "standard" coordinate system $V_{\theta}^{\prime}>0$, if it is directed to the north [3]); $V_{\lambda}^{\prime}>0$ if velocity is directed to the east, $\bar{V}_{\lambda}(\theta)$ - zonal large-scale flow caused by the inhomogeneous heating of polar and equatorial regions, $\alpha$ - circulation index (angular velocity of the main zonal flow from the west to the east). Since $V_{r}^{\prime}=0, r$ is constant parameter equal to the Earth's radius $R=6.4 \cdot 10^{6} \mathrm{~m}$.

According to (1.4) condition of velocity solenoidality admits the existence of the current function $\psi=\bar{\psi}(\theta)+\psi^{\prime}(\theta, \lambda, t)$ :

$v_{\theta}^{\prime}=-\frac{1}{R \sin \theta} \frac{\partial \psi^{\prime}}{\partial \lambda}, v_{\lambda}^{\prime}=\frac{1}{R} \frac{\partial \psi^{\prime}}{\partial \theta}, \bar{v}_{\lambda}(\theta)=\frac{1}{R} \frac{d \bar{\psi}}{d \theta}$.

The exact solution for the function $\psi^{\prime}$ is sought using the well known equation $[1,5]$ :

$\frac{\partial \Delta \psi^{\prime}}{\partial t}+\alpha \frac{\partial \Delta \psi^{\prime}}{\partial \lambda}+2\left(\alpha+\omega_{0}\right) \frac{\partial \psi^{\prime}}{\partial \lambda}=0$,

where $\Delta=\frac{1}{\sin \theta} \frac{\partial}{\partial \theta}\left(\sin \theta \frac{\partial}{\partial \theta}\right)+\frac{1}{\sin ^{2} \theta} \frac{\partial^{2}}{\partial \lambda^{2}}$ is the spherical Laplace operator. Since the equation (1.7) is linear, its general solution always can be presented in the form of superposition of the particular solutions. The particular solution $\psi^{\prime}=c P_{n}^{m}(\cos \theta) \exp (-i \omega t+i m \lambda)$, where $c$ is arbitrary constant, $P_{n}^{m}(\cos \theta)$ - associated Legendre polynomial leading to the well known dispersion equation for the frequency of the planetary Rossby waves [1]:

$\omega=\omega_{n}^{m}=\alpha m-\frac{2\left(\alpha+\omega_{0}\right) m}{n(n+1)}$,

where $m$ and $n$ are zonal and meridional wave numbers, $m=0,1,2,3, \ldots, n, \quad n=1,2,3, \ldots,$. Pressure $P$ is easily determined from the Euler equation in the quadrature. These results have numerous applications in dynamic meteorology $[1,4,5,7]$.

In conclusion of this paragraph it should be pointed out that the exact solution (1.5) describes the Earth's atmosphere in the form of a thin homogeneous spherical film in which the disturbed planetary wave propagates along the latitude circles around the Earth; and oscillations of the atmospheric particles occur along the meridian. The amplitude in this wave is determined by the associated Legendre polynomial. Further we will use only the exact solution (1.5) for the upper atmosphere. We consider three-dimensional propagation of the planetary waves $\left(V_{r} \neq 0\right)$ in the spherical coordinate system, which will naturally determine an interaction of the different atmospheric layers at the upward propagation as a separate independent problem and it will not be investigated in this paper. We consider the $E$ and $F$ regions in the upper atmosphere in the approximation (1.5) as non-interacting, thin, rotating homogeneous spherical layers.

\section{OBTAINING OF THE EXACT SOLUTION FOR THE SYSEM (1.2) - (1.4)}

Let's show that there is an exact solution of the system (1.2) - (1.4) in the presence of magnetic field, when the velocity fields $\bar{v}$ and $\bar{U}$ have the form:

$v_{r}^{\prime}=0, \quad v_{\theta}=v_{\theta}^{\prime}(\theta, \lambda, t), \quad v_{\lambda}=\bar{v}_{\lambda}(\theta)+v_{\lambda}^{\prime}(\theta, \lambda, t)$,

$\bar{v}_{\lambda}(\theta)=\alpha R \sin \theta, U_{\theta}=U_{\theta}^{\prime}(\theta, \lambda, t)$,

$U_{\lambda}=\bar{U}_{\lambda}(\theta)+U_{\lambda}^{\prime}(\theta, \lambda, t), \quad \bar{U}_{\lambda}(\theta)=-\alpha_{i} R \sin \theta$.

The parameter $\alpha_{i}$ will be determined below ("sign " - takes into account the rotation of ions from the east to the west). Introducing the flow functions $\psi^{\prime}$ and $\psi_{a}^{\prime}$, from the conditions of solenoidality (1.4), for the components of velocities $\bar{v}$ and $\bar{U}$, we obtain:

$$
\begin{aligned}
& v_{\theta}^{\prime}=-\frac{1}{R \sin \theta} \frac{\partial \psi^{\prime}}{\partial \lambda}, v_{\lambda}^{\prime}=\frac{1}{R} \frac{\partial \psi^{\prime}}{\partial \theta}, \bar{v}_{\lambda}(\theta)=\frac{1}{R} \frac{\partial \bar{\psi}}{\partial \theta}, \\
& U_{\theta}^{\prime}=-\frac{1}{R \sin \theta} \frac{\partial \psi_{a}^{\prime}}{\partial \lambda}, U_{\lambda}^{\prime}=\frac{1}{R} \frac{\partial \psi_{a}^{\prime}}{\partial \theta}, \bar{U}_{\lambda}(\theta)=\frac{1}{R} \frac{\partial \bar{\psi}_{a}}{\partial \theta}
\end{aligned}
$$

from the expressions (2.2) and (1.3) it follows that the magnetic field has only vertical component

$$
\frac{H_{r}}{\alpha \rho}=\frac{H_{0 r}(\theta)}{\alpha \rho}+\frac{h_{r}(\theta, \lambda, t)}{\alpha \rho}=\operatorname{rot}_{r} \mathbf{U}_{0}+\operatorname{rot}_{r} \mathbf{U} .
$$

Omitting the primes from the equations (1.2) and (1.3), we obtain the system of equations for the functions $\psi$ and $\psi_{\alpha}$ :

$$
\begin{aligned}
& \frac{\partial \Delta \psi}{\partial t}+\alpha \frac{\partial \Delta \psi}{\partial \lambda}+2\left(\alpha+\omega_{0}\right) \frac{\partial \psi}{\partial \lambda}+2 \Omega_{e} \frac{\partial \Delta \psi_{a}}{\partial \lambda}=0 \\
& \frac{\partial \Delta \psi_{a}}{\partial t}+\alpha \frac{\partial \Delta \psi_{a}}{\partial \lambda}-2\left(\alpha_{i}+\Omega_{i E}\right) \frac{\partial \psi}{\partial \lambda}-\delta 2 \Omega_{e} \frac{\partial \Delta \psi_{a}}{\partial \lambda}=0
\end{aligned}
$$

where $\Omega_{i E}=\mu \omega_{i E}, \Omega_{e}=\left(c / c_{e}\right)^{2} \omega_{e E}, \omega_{i E}=e H_{E} / M c$, $\omega_{e E}=e H_{E} / m c$ are cyclotron frequencies of ions and electrons at the equator, respectively. From the identity

$$
\begin{aligned}
& \frac{H_{0 r}}{\alpha \rho}=-2 \Omega_{i E} \cos \theta=\operatorname{rot}_{r} \mathbf{U}_{0}=\frac{1}{R \sin \theta} \frac{\partial}{\partial \theta}\left(\sin \theta \bar{U}_{\lambda}(\theta)\right)= \\
& =\frac{1}{R \sin \theta} \frac{\partial}{\partial \theta} \sin \theta \frac{1}{R} \frac{d \bar{\psi}_{a}}{d \theta}=-\frac{\alpha_{i}}{\sin \theta} \frac{\partial}{\partial \theta} \sin ^{2} \theta
\end{aligned}
$$


it follows that $\alpha_{i}=\Omega_{i E}$. Therefore, the coefficient of the third term in the equation (2.4) takes on the following form: $\alpha_{i}+\Omega_{i E}=2 \Omega_{i E}=\mu \omega_{i p}=\Omega_{i}, \omega_{i p}=\left(e H_{p} / M c\right)>0$ is the cyclotron frequency of ions at the pole. If in the equations (2.3) and (2.4) we will pass on to the local Cartesian coordinate system ("standard" system $[1,7]$ ), taking into account $\alpha<\omega_{0}, \alpha_{i}=0$ and using the approximation formulae $\frac{1}{R \sin \theta} \frac{\partial}{\partial \lambda} \approx \frac{\partial}{\partial x} ; \frac{1}{R} \frac{\partial}{\partial \theta} \approx-\frac{\partial}{\partial y}$,

$\Delta \approx R^{2}\left(\frac{\partial^{2}}{\partial x^{2}}+\frac{\partial^{2}}{\partial y^{2}}\right)=R^{2} \Delta_{s}, \Delta_{s}=\frac{\partial^{2}}{\partial x^{2}}+\frac{\partial^{2}}{\partial y^{2}}$,

we obtain the system of equations obtained in [7]. As an example let's show validity of such transformation for the Rossby waves. Neglecting the action of the geomagnetic field and assuming that $\bar{v}_{\lambda}(\theta)=\bar{u}=$ const, from the equation (1.11) taking into account $\alpha=\bar{u} / R \cdot \sin \theta$ we find

$$
\left(\frac{\partial}{\partial t}+\frac{\bar{u}}{R \sin \theta} \frac{\partial}{\partial \lambda}\right) R^{2} \Delta_{s} \psi+\frac{2 \bar{u}+2 \omega_{0} R \sin \theta}{R \sin \theta} \frac{\partial \psi}{\partial \lambda}=0 .
$$

Neglecting the small term $2 \bar{u} / R^{2}$ we will obtain the well known Rossby equation and the dispersion equation for the phase velocity in a "standard" coordinate system [4]:

$\left(\frac{\partial}{\partial t}+\bar{u} \frac{\partial}{\partial x}\right)\left(\frac{\partial^{2} \psi}{\partial x^{2}}+\frac{\partial^{2} \psi}{\partial y^{2}}\right)+\beta \frac{\partial \psi}{\partial x}=0$,

$c_{p h}=\bar{u}-\frac{\beta}{4 \pi^{2}} L^{2} \frac{d^{2}}{L^{2}+d^{2}}, \quad$ where $\quad L=2 \pi / k_{x} \quad$ is the wavelength, $d=2 \pi / k_{y}$ wave width, $\beta=-\partial 2 \omega_{0 z} / \partial y=$ $2 \omega_{0} \sin \theta / R$ - Rossby parameter. The approximate calculation of the Earth's curvature lies in the fact that the Rossby parameter $\beta$ is considered constant however $\beta$ depends on $\theta$. The expression $\partial \beta / \partial(R d \theta)$ has the order $\omega_{0} / R^{2}$ and its variation can be practically always neglected [15]. Similarly the results for the magnetogradient planetary waves are easily obtained from the set of equations (2.3) and (2.4) in $\beta$ - approximation. The advantage of $\beta$ approximation, i.e., approximate replacement of spherical coordinates with Cartesian coordinates: $d x \approx R \sin \theta \cdot d \lambda$, $d y \approx-R \cdot d \theta, d z \approx d r$ lies in the fact that the equations of motion in the spherical and Cartesian coordinate systems have the same form. This replacement procedure of variables strongly simplifies the problem of investigation of the dynamics of large-scale processes in the atmosphere, since in this case the problem is reduced to the investigation of motion on the rotating homogeneous plane (but not to the rotating spherical film). Solution of the problem in the "standard" coordinate system is sought in the form of the plane waves having constant amplitude and the wave numbers $k_{x}, k_{y}, \quad\left(\psi^{\prime}=A \exp \left(-i \omega t+i k_{x} x+i k_{y} y\right)\right)$. In this approximation the Earth's sphericity is considered only by the Rossby parameter $\beta=-\partial 2 \omega_{o z} / \partial y=2 \omega_{0} \sin \theta / R$.

In the case under consideration the equations (2.3) and (2.4) solve the problem of the planetary waves in the rotating ionosphere. Knowing $\psi$ and $\psi$ we can find the components of small perturbations $v_{\theta}^{\prime}, v_{\lambda}^{\prime}, h_{r}$ superimposing on the main zonal motion of ionospheric medium $\bar{v}_{\lambda}(\theta)$. After this, the pressure $P$ is determined by quadrature from the equation of motion of the ionospheric medium. Since in the system (2.3) and (2.4) coefficients do not depend on the variables $\lambda$ and $t$, we will seek the solution of the system in the form of zonal waves $\psi$, $\psi_{a} \sim \exp (-i \omega t+i m \lambda)$. Substituting these expressions for $\psi$ and $\psi_{a}$ in the equations (2.3) and (1.4) and excluding $\psi_{a}$, we obtain one equation for the current function $\psi$ :

$\Delta \psi+\left\{\frac{2\left(\alpha+\omega_{0}\right) m}{\sigma}+\frac{2 \Omega_{e} m 2 \Omega_{i} m}{\sigma\left(\sigma-\delta 2 \Omega_{e} m\right)}\right\} \psi=0$,

where $\sigma=\alpha m-\omega$.

Particular solution $\psi=c P_{n}^{m}(\cos \theta) \exp (-i \omega t+i m \lambda)$ of the equation (2.5) is restricted on a whole sphere only in case if the equality is fulfilled:

$\frac{2(\alpha+\omega) m}{\sigma}+\frac{2 \Omega m 2 \Omega m}{\sigma(\sigma-\delta 2 \Omega m)}=n(n+1)$,

where $n$ is any integral number, $m=0,1,2,3, \ldots, n$.

Introducing the notations $\frac{2\left(\alpha+\omega_{0}\right) m}{n(n+1)}=\omega_{R}, \frac{2 \Omega_{i} m}{n(n+1)}=\omega_{R}^{\prime}$ and taking into account that $\frac{\omega_{R}}{2 \Omega_{e} m}<<1$, we will obtain the general dispersion equation for the frequencies of the hydrodynamic and electromagnetic planetary waves in the rotating ionosphere:

$\sigma^{2}-\delta 2 \Omega_{e} m \sigma+2 \Omega_{e} m\left(\delta \omega_{R}-\omega_{R}^{\prime}\right)=0$,

For the $E$-region of ionosphere $(\delta=1)$ the expression (2.7) takes on the following form:

$\frac{\sigma}{2 \Omega_{e} m}+\frac{\omega_{R}-\omega_{R}^{\prime}}{\sigma}=1$

hence, for the high frequencies $\sigma \gg>1(\omega>>\alpha m)$ from (2.8) we obtain:

$\omega_{+}=-2 \Omega_{e} m=\frac{c H_{p}}{4 \pi e N R^{2}} m,(e<0)$.

For low frequencies $\sigma<<1$ we have

$\omega_{-}=\alpha m-\frac{2\left(\alpha+\omega_{0}-\Omega_{i}\right) m}{n(n+1)},\left(\Omega_{i}>0\right)$. 
For the intermediate frequencies, taking into account $\omega_{R}^{\prime}>\omega_{R}$ and $\omega>\alpha m$, the dispersion equation (2.8) can be rewritten in the following form:

$$
\omega_{n}= \pm \frac{V_{a}}{R} \frac{1}{\left(1+\frac{2 \Omega_{e} m}{\omega}\right)^{1 / 2}} \frac{m}{\sqrt{n(n+1)}},
$$

where $V_{a}=H_{p} / \sqrt{4 \pi \rho}$ is the velocity of slow MHD wave at the pole. Thus, three types of the very long planetary waves should exist in the spherical coordinate system in the $E$-region of the ionosphere: ultra-low-frequency waves from two days to two weeks and longer, slow $10 \div 100 \mathrm{~m} \cdot \mathrm{s}^{-1}$ Rossby type planetary waves, caused by the Earth's rotation $\left(\omega_{0} \neq 0\right)$ and geomagnetic field $\left(H_{p} \neq 0\right)$. They have hydrodynamic nature (formula (2.10)); two fast (1 $\mathrm{km} \cdot \mathrm{s}^{-1}$ and higher) high-frequency (from tens of seconds to several hours) planetary waves, caused by the vortex electrical field (formula (2.9)) and electromagnetic Ampere force $F_{a}$ (formula (2.11)) have electromagnetic nature [6-9]. Physically these waves represent oscillations of the magnetized electrons (formula (2.9)), ions and neutral particles (formula (2.10)) and oscillation of the $E$-region as a whole (formula (2.11)). The dispersion of electromagnetic planetary waves (2.11) increases when frequency decreases and disappears for the high frequencies. In the $F$-region of ionosphere $(\delta=0)$, as it follows from the general dispersion equation (2.7), only one fast electromagnetic planetary wave is generated:

$\omega_{n}= \pm \frac{V_{a}}{R} \frac{m}{\sqrt{n(n+1)}}$.

Formulae (2.9) - (2.12) can be substantially simplified taking into account that between the wavelength $\lambda$ and zonal wave number $m$ there is a simple link $m=2 \pi R \sin \theta / \lambda=k R \sin \theta$ (showing how many waves are located along the each circle of the latitudes by the radius $R \sin \theta)$, introducing angular velocity $(d \lambda / d t)=\omega / \mathrm{m}$ and phase velocity of waves along the circles of the latitudes $c_{\varphi}=R \sin \theta(d \lambda / d t)$ we obtain the formulae convenient for the numerical analysis: for the $E$ - region:

$$
\begin{aligned}
& c_{H}=c_{p h+}=\frac{c H_{p} \sin \theta}{4 \pi e N R}, \\
& c_{R}^{\prime}=c_{p h-}=\left\{\alpha-\frac{2\left(\alpha+\omega_{0}-\Omega_{i}\right)}{n(n+1)}\right\} R \sin \theta, \\
& c_{n}= \pm \frac{V_{a} \sin \theta}{\sqrt{n(n+1)}} \frac{1}{\left(1+\frac{2 \Omega_{e} m}{\omega}\right)^{1 / 2}},
\end{aligned}
$$

and for the $F$ - region

$$
c_{n}= \pm \frac{V_{a} \sin \theta}{\sqrt{n(n+1)}} .
$$

\section{NUMERICAL ANALYSIS OF THE PARAMETERS OF PLANETARY WAVES IN THE IONOSPHERE AND DISCUSSION OF THE OBTAINED RESULTS}

At the estimation of the spectrum of frequencies of the magnetogradient planetary waves in the $E$ and $F$-regions of ionosphere we will use the well known middle-latitude models of neutral atmosphere and ionosphere. Dispersion equation (2.7) formally links these layers through the parameter $\delta$; description of the wave processes in $E$ - region corresponds, and in $F$ - region $\delta=0$.

Numerous observations show [17-21] that during earthquakes, man-made explosions, magnetic storms, launching of spacecrafts, worldwide networks of ionospheric and magnetic observatories (located approximately along one latitude) in the $E$-region of ionosphere besides the well known wave modes the large-scale $\left(\lambda \sim 10^{3} \div 10^{4} \mathrm{~km}\right)$ ionospheric wave disturbances of electromagnetic nature $((\partial \mathbf{h} / \partial t) \neq 0)$ are clearly registered propagating along the parallel around the Earth with high (supersonic) speeds (higher than $1 \mathrm{~km} \cdot \mathrm{s}^{-1}$ ) and having periods from several minutes to several hours. In the same region of the upper atmosphere are also observed large-scale, long-period (from two days to two weeks and longer) wave disturbances of hydrodynamic nature $((\partial \mathbf{h} / \partial t) \approx 0)$ (their velocity is of the order of the velocity of the ionospheric winds). Unlike the very long planetary Rossby waves (propagating mainly westward) they propagate to the east and generating the electric currents lead to the essential pulsations of the geomagnetic field (to $45 \mathrm{nT}$ and higher).

The observed parameters of the fast waves in the $E-$ region of ionosphere are sufficiently consistent with the parameters of the well studied large-scale middle-latitude long-period oscillations (MLO). It has been established that the phase velocities of MLF for the day and nighttime conditions differ almost by an order and higher and have insignificant altitude curve [8,21].

Regular observations at the middle latitudes of the $F-$ region of ionosphere revealed large-scale, fast $\left(5-50 \mathrm{~km} \cdot \mathrm{s}^{-1}\right)$ magnetoionospheric wave disturbances (MIWD) of electromagnetic nature with the period from several seconds to tens of minutes and longer. An important role of the geomagnetic field in forming these electromagnetic, lowfrequency oscillations is emphasized in this term. Unlike the MLO, the characteristic parameters of MIWD strongly vary with height and depending on the magnetic activity of the Sun [18]. The amplitude of geomagnetic pulsations in these disturbances vary from several tens to hundreds $\mathrm{nT}$ and the ionospheric parameters up to $0,5-30 \%$.

Let us show that the observed wave disturbances of planetary scale in the $E$ and $F$ - regions of ionosphere are the response of a new branch of the own oscillations of ionospheric resonator [7] and the features of the observed disturbances can be described well by the above obtained formulae (2.9) - (2.14). Numerical calculations of the parameters of fast and slow planetary waves were carried out for comparison of the obtained theoretical results by the 
above mentioned formulae with the observed values of the parameters of MLO, MIWD and the slow long-period wave disturbances. The models of ionosphere and neutral atmosphere [22] were used for the periods of low and high activity of the Sun (with the values of exospheric temperatures $T_{\text {exosphere }}=600^{\circ} \mathrm{K}$ and $2600^{\circ} \mathrm{K}$ ) in the range of the heights between $90-500 \mathrm{~km}$ at $\theta=45^{\circ}$, at different times in twenty-four hours zonal $(m=5)$ and meridional $(n=6.7)$ numbers.

Since the general expression of Hall conductivity $\sigma_{2}$ depends on the sign of a charge [23] then electronic conductivity should be subtracted from the ionic conductivity at its calculation. Calculations showed that beginning from $150 \mathrm{~km}$ and higher they cancel each other and Hall conductivity reduces to zero. Therefore further by the Hall region will be meant the region of the heights $90 \div 150 \mathrm{~km}$. The calculated value of the fast and slow planetary waves for this region of the upper atmosphere will be compared with the observed parameters of large-scale disturbances in the $E$-region of ionosphere. At calculation of the parameters of fast planetary waves in the $F$-region we will limit ourselves to the heights from 200 up to $500 \mathrm{~km}$. The transition region of ionosphere is $150 \div 200 \mathrm{~km}$, which we will not touch on as it requires special consideration.

Below as an example we will present only some results of numerical calculations of the considered waves for the middle-latitude ionosphere. Let's begin investigation from the calculation of parameters of the fast magnetogradient planetary waves in the $F$ - region of ionosphere. Using formulae (2.12) and (2.14) for the low ( $\left.T_{\text {exosphere }}=600^{\circ} \mathrm{K}\right)$ and high $\left(T_{\text {exosphere }}=2600^{\circ} \mathrm{K}\right)$ activities of the Sun the values of parameters of the fast planetary waves in the range of the heights 200-500 km were calculated. The calculations showed that at $T_{\text {exosphere }}=600^{\circ} \mathrm{K}, n=6$ and 7 the phase velocity between the heights $200-500 \mathrm{~km}$ varies from 10 to $50 \mathrm{~km} \cdot \mathrm{s}^{-1}$, and oscillation period $T_{n}=2 \pi / \omega_{n}$ from 105 to $5 \mathrm{~s}$; at $T_{\text {exosphere }}=2600^{\circ} \mathrm{K}$ the phase velocity varies from 5 to $20 \mathrm{~km} \cdot \mathrm{s}^{-1}$, and oscillation period from 200 to $40 \mathrm{~s}$. The strong dependence of the parameters $c_{n}$ and $T_{n}$ on the exospheric temperature (the decrease of $c_{n}$ and increase of $T_{n}$ ) can be explained "by the "inflation" of atmosphere at high activity of the Sun [24] and by lifting of "heavy" particles from the lower layers of the ionosphere, which at the observed heights leads to the increase of density $\rho$ of the upper atmosphere. The periods and phase velocities of the fast waves correlate well with the observed parameters of the middle-latitude large-scale disturbances of MIWD generated in the $F$-region of ionosphere during the strong (with magnitude $7 \leq M \leq 8$ ) earthquakes and magnetic storms [18,25]. An increase of MIWD displacement velocity with height found experimentally by these authors directly follows from the analytical formula (2.14) for $c_{n}$.
Calculations of the parameters of the fast magnetogradient planetary waves in the $E$-region of the ionosphere in the range of the heights $90-150 \mathrm{~km}$ were done using the formulae (2.9) and (2.13) (first formula). Calculations showed that the influence of exospheric temperature on the values $c_{H}$ and $T_{H}$ is insignificant, but the influence of the perturbed geomagnetic field $h_{r}$ is essential. Since the values of $c_{H}$ and $T_{H}$ depend only on the electron density $N$, which in the course of twenty-four hours varies by an order and higher, the daytime values of these parameters strongly differ from the nighttime ones. Estimations show that the value $c_{H}$ in the range of the heights $90-150 \mathrm{~km}$ of the order of $0,1-0,5 \mathrm{~km} \cdot \mathrm{s}^{-1}$ and $0,5-5 \mathrm{~km} \cdot \mathrm{s}^{-1}$ for the day and nighttime conditions, respectively. At the observed heights if $\lambda=5600 \mathrm{~km}$, the day and night values of the periods vary from $2 \cdot 10^{4} \mathrm{~s}$ to $4 \cdot 10^{3} \mathrm{~s}$ and from $4 \cdot 10^{3} \mathrm{~s}$ to $3 \cdot 10^{2} \mathrm{~s}$, respectively. With increase of the wavelength the numerical values of the periods $c_{H}$ - waves increase proportionally. With displacement of the electrons $\xi_{\theta e}$ equal to $0,5-2 \mathrm{~km}$ the perturbation of magnetic field $h_{r}$ varies in the range of 5-150 nT, which is in agreement with the estimation [9]. The parameters of $c_{H}$ - waves correlate well with the parameters of MLO in the middle-latitude of the $E$-region of ionosphere under the man-made influences on the ionosphere (industrial explosions, launching of spacecrafts) $[9,26]$ and also during the earthquakes and magnetic storms [25]. The observed mechanism of high velocity displacements of MLO - waves in nighttime conditions and strong decrease of their values in daytime ionosphere $[18,21]$ directly follows from the first formula (2.13).

Let's proceed to the slow Rossby type planetary waves (formula (2.10) and the second formula (2.13)) in the $E$ region of ionosphere. Calculations show that the velocities $C_{R}^{\prime}$ at $T_{\text {exosphere }}=600^{0} \mathrm{~K}, \lambda=5600 \mathrm{~km}, \alpha=0$ in the range of the heights $90-150 \mathrm{~km}$ vary from $-41 \mathrm{~m} \cdot \mathrm{s}^{-1}$ to $2 \mathrm{~km} \cdot \mathrm{s}^{-1}$ in daytime and from $-41 \mathrm{~m} \cdot \mathrm{s}^{-1}$ to $15 \mathrm{~m} \cdot \mathrm{s}^{-1}$ - at nighttime. Minus sign indicates the direction of phase velocities from the east to the west and sign plus - from the west to the east. The dependence of $C_{R}^{\prime}$ - waves on the exospheric temperature is caused by the fact that the planetary wave in the parameter $\Omega_{i}$ contains ionization degree $\eta=N / N_{n}$ which as in the $c_{n}$ - waves with increase of a height leads to an increase of the phase velocities. Calculations also show that in daytime at the height of 115 $\mathrm{km}$ frequencies $\omega_{0}$ and $\Omega_{i}$ compensate each other. In the nighttime ionosphere this level is located at the height of 150 $\mathrm{km}$. Calculation of the periods $T_{R}^{\prime}$ show that at $T_{\text {exosphere }}=600^{\circ} \mathrm{K}$ in the range of the heights of $90-150 \mathrm{~km}$ vary from 14 up to 8 days, and at $T_{\text {exosphere }}=2600^{\circ} \mathrm{K}$ - from 14 up to 2 days. The disturbance of geomagnetic field in 
these waves reaches from two to several tens of $n T$. The parameters of Rossby type waves correlate well with the parameters of planetary waves in the $E$-region of ionosphere observed at the middle heights in any season of a year [18].

Physically the mechanism of the excitation of free zonal oscillations $c_{n}$ - waves in the $F$ - region of ionosphere $(\delta=0)$ simply follows from the simplified equations (1.2) (1.3) in the "standard" coordinate system of [7].

$i \omega V_{y}=\frac{\beta_{H}}{4 \pi \rho} h_{z}, i \omega h_{z}=-\beta_{H} V_{y}$,

where $\beta_{H}=\frac{\partial H_{0 z}}{\partial y}=-\frac{1}{R} \frac{\partial}{\partial \theta}\left(-H_{p} \cos \theta\right)=-\frac{H_{p}}{R} \sin \theta$ is the Rossby "magnetic" parameter. Indeed, introducing the displacement $V_{y}=d \xi_{y} / d t=i \omega \xi_{y}$ and specific quasi-elastic force $f=\beta_{H} h_{z} / 4 \pi \rho=-\beta_{H}^{2} \xi_{y} / 4 \pi \rho$ from (3.1) we obtain the equation of free oscillations for linear oscillator.

$$
\begin{aligned}
& \frac{d^{2} \xi_{y}}{d t^{2}}+\frac{\beta_{H}^{2}}{4 \pi \rho} \xi_{y}=0, \omega_{0}^{2}=\frac{\beta_{N}^{2}}{4 \pi \rho}=\frac{k}{\rho} \\
& \omega_{n}= \pm \frac{V_{a}}{R} \sin \theta .
\end{aligned}
$$

In this case from the "freezing-in" condition $h_{z}=-\beta \xi_{y} \approx \frac{H_{0 z}}{R} \xi_{y}$ (second equation of the system (3.1)) follows that any transverse displacement $\xi_{y}$ of neutral particle in the $F$-region of ionosphere generates tension of the lines of force of the geomagnetic field $H_{0 z}$ in the ionospheric plasma due to the collisional processes. As a result a component proportional to $\xi_{y}$ appears in the magnetic field $H_{0 z}$ being the cause of generation of quasielastic force $f=-\beta \xi / 4 \pi \rho=-k \xi / \rho$. Here the value $k=\beta_{i}^{2} / 4 \pi \rho$ can be termed a coefficient of electromagnetic elasticity of the ionospheric medium.

In the $E$-region of ionosphere $(\delta=1)$ where the Hall effect plays a decisive role, it is necessary to consider ionospheric medium as three-component fluid [27]. In this region of the upper atmosphere the ions are completely entrained by the neutral particles $\mathbf{V}_{i}=\mathbf{V}_{n}$ [24]. Then from the induction equation (1.3), introducing explicitly the velocity of electrons by the formula $\mathbf{V}_{e}=\mathbf{V}_{i}-(\mathbf{j} / e N)=\mathbf{V}_{n}-(\mathbf{j} / e N)$, in the old variables we obtain the condition of complete "freezing-in" of the geomagnetic field $H_{0}$ in the electronic component:

$$
\frac{\partial \mathbf{h}}{\partial t}=\operatorname{rot}\left[\mathbf{V}_{e} \mathbf{H}_{0}\right]
$$

Since the ions move with the velocity of neutral particles (with wind velocity) they can be considered as fixed in comparison with the velocity of electrons $\mathbf{V}_{e}$ [24]. Then excluding from (3.3) using the formula $\mathbf{V}_{e} \approx-\mathbf{j} / e N=-c \operatorname{rot} \mathbf{h} / 4 \pi e N$ we obtain the closed induction equation for the variable $\mathbf{h}$

$\frac{\partial \mathbf{h}}{\partial t}=\frac{c}{4 \pi e N}\left[\operatorname{rot} \mathbf{h} \cdot \mathbf{H}_{0}\right]$,

in the variables $h_{z}$ or $V_{e y}$ the last expression can be rewritten in the following form:

$\frac{\partial h_{z}}{\partial t}=-\frac{c \beta_{H}}{4 \pi e N} \frac{\partial h_{z}}{\partial x}$ or $\frac{\partial V_{e y}}{\partial t}=-\frac{c \beta_{H}}{4 \pi e N} \frac{\partial V_{e y}}{\partial x}$.

For the zonal waves $h_{z}, V_{e y} \sim \exp \left(i \omega t-i k_{x} x\right)$ the phase velocity will take on the following form

$$
c_{p h}=\frac{\omega}{k_{x}}=c_{H}=\frac{c}{4 \pi e N} \frac{\partial H_{0 z}}{\partial y} .
$$

Thus, in the $E$-region of ionosphere in the fast planetary waves oscillations occur practically with the fixed ions and neutral particles and oscillate only electrons frozen in the lines of force of geomagnetic field. At the same time the induced magnetic field $\mathbf{h}$ and the vortex electrical field rot $E_{\text {vort }} \neq 0$ are generated. Indeed, introducing displacement of electrons $V_{e y}=\partial \xi_{e y} / \partial t$, from the second equation (3.5) we obtain the equation of linear oscillator $\frac{d^{2} \xi_{e y}}{d t^{2}}+\omega_{0}^{2} \xi_{e y}=0 \quad$ with eigenfrequency $\omega_{0}^{2}=\omega_{H} \omega$, $\omega_{H}=\frac{c \beta_{H} k_{x}}{4 \pi e N}=\frac{c H_{p} \sin \theta}{4 \pi e N} k_{x}$.

By comparing expression (3.6) with the phase velocity of the small-scale whistlers (helicons) $c_{n}=c k_{z} H_{0 z} / 4 \pi e N$ [27] it should be noted that the physical reason of oscillations of both eigenmodes is similar - oscillate the electrons frozen in the lines of force of geomagnetic field. The difference is that for the waves of the whistlers the phase velocity depends on the vertical component of geomagnetic field $H_{0 z}$ and vertical wave number $k_{z}$, while for the planetary $c_{H}$-waves on the gradient of geomagnetic field $\frac{\partial H_{0 z}}{\partial y}=-\frac{1}{R} \frac{\partial H_{0 z}}{\partial \theta}$, which naturally determines large-scale $c_{H^{-}}$waves. Electromagnetic interaction of the internal vortical electrical field $\mathbf{E}$ with the ionospheric plasma in the $E$-region when $\mathbf{V}_{\mathbf{e}}=\mathbf{V}_{\mathbf{D}}=c\left[\mathbf{E} \mathbf{H}_{\mathbf{0}}\right] / H_{0}^{2}>\mathbf{V}=\mathbf{V}_{\mathbf{i}}$, can be termed fast processes for planetary scale perturbations, and the method of determining the eigenfrequencies - as induction approximation $(\partial \mathbf{h} / \partial t) \neq 0$ of magneto-hydrodynamics $[24,28]$. As a result under the influence of internal electrical field $\mathbf{E}$ electronic component moves with the electric drift velocity $\mathbf{V}_{e}=\mathbf{V}_{D}$ in the $E$-region of ionosphere and the ionic component moves together with the neutral wind 
$V_{i x}=V_{n x}$. Hence it follows that the generable internal (vortical) electrical field $\mathbf{E}$ orthogonal with respect to the geomagnetic field $H_{0 z}$ will have both zonal $E_{x}$ and meridional $E_{y}$ components. At the same time the electrons under the influence of the $E_{y}$ component, generated by Lorentz force $F_{y}=-e V_{x} H_{0 z} / c=-e E_{y}$, will move in the meridional direction (Hall effect), and ions - in zonal direction. The direction of the summed electric current will depend on the relation between Pedersen and Hall conductivities. Since the Hall conductivity substantially exceeds Pedersen conductivity in this interval of the upper atmosphere $(80 \div 150 \mathrm{~km})$, the direction of a summed current will be close to the meridional direction. Thus, in our task the quasineutrality of ionospheric plasma in the $E$-region does not mean that the effect of the charges separation is neglected. It is considered indirectly, in particular, by means of introducing the internal electrical field $\mathbf{E}$ mainly caused by the Hall effect.

For the $E$-region of ionosphere $(\delta=1)$ adding equations (1.1) and taking into account that $\mathbf{h} \ll \mathbf{H}_{0}$, we will obtain the condition of "partial" freezing-in of the geomagnetic field:

$h e l m\left(\operatorname{rot} \mathbf{V}+2 \boldsymbol{\omega}_{\mathbf{0}}+\frac{\mathbf{H}_{\mathbf{0}}}{\alpha \rho}\right)=0$,

which is the closed equation for velocity $\mathbf{V}$. The exact solution (3.7) is Rossby type "ultra-slow" planetary waves propagating in the $E$-region with the phase velocity: $c_{R_{0}}^{\prime}=c_{p h}=\omega / k_{x}=-\left(\beta+\beta_{H}\right) / k_{x}^{2}$,

where $\beta=\partial\left(2 \omega_{0 z}\right) / \partial y$ - the usual Rossby parameter. Since $\omega_{0 z}$ and $H_{0 z}$ have opposite directions $\left(\omega_{0 z}>0, H_{0 z}<0\right)$, phase velocity of slow Rossby type waves $c_{R_{0}}^{\prime}$ will be less than phase velocity of the usual Rossby waves $\left(c_{R}=-\beta / k_{x}^{2}\right)$. In these waves $(\partial \mathbf{h} / \partial t) \approx 0$, i.e. $\operatorname{rot} \mathbf{E} \approx 0$ and the formula $\mathbf{E}=-\operatorname{grad} \phi$ together with the expression $\operatorname{rot}\left[\mathbf{V H}_{\mathbf{0}}\right]=0$ uniquely determines the electrostatic field of polarization $\mathbf{E}_{d}$ (dynamo field), which is generated in the $E$-region of ionosphere by the wind mechanism.

$\mathbf{E}=-\nabla \phi=-\frac{1}{c}[\mathbf{V} \mathbf{H}]$.

Introducing the displacement $\xi_{y}$ and quasi-elastic force $f=-\frac{\beta+\beta_{H}}{k_{x}} \frac{\omega \rho}{\rho} \xi_{y}=-\frac{k}{\rho} \xi_{y}$, where $k=\frac{\beta+\beta_{H}}{k_{x}} \omega \rho$, from (3.7) it is easy to obtain the equation of free oscillations for the Rossby type waves $c_{R_{0}}^{\prime}$ :

$\frac{d^{2} \xi_{y}}{d t^{2}}+\omega_{0}^{2} \xi_{y}=0, \omega_{0}^{2}=-\frac{\beta+\beta_{H}}{k_{x}} \omega$.
In the monograph by Holton [29] is given the diagram of propagation of the very long (500 km and longer) planetary Rossby wave in the troposphere for the northern hemisphere $\left(2 \omega_{0 z}>0\right)$ from which it is apparent that the particles in planetary wave oscillate along the meridian, and a wave propagates along the parallel to the west. At the same time motion of atmospheric particles in the wave is such, that nonzero vorticity is generated characterized by the regions of low and high pressures, which under the conditions of geostrophic balance correspond to the tropospheric weatherforming cyclones and anticyclones. Hence follows the very important conclusion for the dynamics of the upper atmosphere. The experimental detection of the planetary waves at the ionospheric levels is the first physical sign of the presence of large-scale vortices (cyclones, anticyclones), which play an important role in weather-forming processes in the ionosphere $[3,15]$.

Vertical vortex (cyclone or anticyclone) in the ionosphere is generated mainly by the $\beta^{\prime}$ - effect [4]

$\frac{\partial \operatorname{rot} \mathbf{V}}{\partial t}=-\beta^{\prime} V_{y}$

i.e. by the meridional winds $V_{y}$ and with allowance for the changes of the angular velocity of the Earth's rotation and geomagnetic field with the latitude $\beta^{\prime}=\beta+\beta_{H}$. At present in the dynamic meteorology this concept of generation of weather-forming vortices in troposphere (cyclones, anticyclones) is conventional. Together with the effect of baroclinity it is necessary to take into account the action of relief of the underlying surface and turbulent friction in the troposphere and action of the force of ionic friction - in the ionosphere; however, $\beta^{\prime}$ - effect remains the determining factor in the mechanism of generation of planetary scale vortex motion in the upper atmosphere of the Earth.

Similarly (3.9) from the equations (1.3) in [12] was obtained important relation revealing mechanism of generation of vortex electrical field in the ionosphere:

$\frac{\partial \operatorname{rot}_{z} U}{\partial t}=\operatorname{rot} \mathbf{E}_{v o r t}=-\frac{\beta^{\prime}}{c}\left(1-\frac{c_{p h}}{c^{\prime}{ }_{R}}\right) V_{y}$.

Similarly to the equation (3.3), when large-scale vortex (cyclone, anticyclone) is generated by the $\beta^{\prime}$ - effect and meridional winds, from (3.10) it follows that the vortex component of the large-scale internal electrical field in the ionosphere should be generated by the $\beta^{\prime}$ - effect, meridional winds $V_{y}$ and fast magnetogradient perturbations in the ionosphere $\left(c_{\varphi}\right)$. From (3.10) it was shown that the relation of the vortex $E_{y, \text { vort }}$ and the dynamo field $E_{d, x}=-c^{-1} V_{y} H_{0 z}$ can be expressed by the important formula:

$\frac{E_{y, v o r t}}{E_{d, x}} \approx \frac{\lambda}{2 \pi R} \frac{c_{p h}}{c_{R}^{\prime}}$, 
which in the $E$-region for the fast planetary wave $\mathrm{c}_{p h}=\mathrm{c}_{H} \approx 2 \mathrm{~km} \cdot \mathrm{s}^{-1},\left(E_{y, \text { vort }} / E_{d, x}\right) \approx 3,\left(E_{y, \text { vort }} / E_{d, x}\right)=15$. From these estimations it clearly follows that for the fast magnetogradient planetary waves the generable vortex electrical field must play a fundamental role in the electrodynamics of the ionosphere. For the slow planetary waves, when $c_{\varphi}=c_{R}^{\prime}$, from (3.10) it follows that the vortical electrical field disappears and in this case only electrostatic dynamo field $\mathbf{E}_{d}$ is generated by the wind mechanism in the ionosphere. The role of curvature of the lines of force of the geomagnetic field $\mathbf{H}_{0}$ with propagation of very long magnetogradient planetary waves was taken into account in [7]. The analysis showed that the real calculation of curvature of the lines of force of geomagnetic field leads to the fundamental result: magnetogradient planetary waves (2.9) - (2.14) propagate with different phase velocities in the western and eastern directions.

\section{CONCLUSION}

The formulae (2.13) and (2.14) in the spherical coordinate system solve the task of planetary waves in rotating ionosphere.

In summary it is can be concluded that the ionosphere, as a resonator, in the MHD approximation (taking into account compressibility and temperature stratification) is described by the closed system of differential equations of the eighth time order. Equations (1.2) and (1.3) give six scalar equations for $\mathbf{v}$ and $\mathbf{h}$. For the density $\rho$ and pressure $P$ we have two more scalar equations (equation of continuity and energy for the polytropic processes) [3]. Therefore at small perturbations ionosphere should have eight eigenfrequencies: two frequencies $\omega_{1,2}$ of the acoustic branch include ordinary sound, magnetic sound, caused by the elasticity of geomagnetic lines of force and its limiting case - helicons (whistlers); two frequencies of the internal gravity waves $\omega_{3,4}$; one frequency $\omega_{5}$ planetary Rossby waves; two frequencies of slow Alfvén type MHD waves $\omega_{6,7}$ caused by the tension of the lines of force of the geomagnetic field by the limiting case which are the slow cyclotron waves of ions $\eta \omega_{i}$ and discovered in [7] the eighth eigenfrequency $\omega_{8}$. In the $E$-region of ionosphere in the electronic component $\omega_{8}=\omega_{H}$; in the ionic component $\omega_{8}=\omega_{R}^{\prime}$ and finally in the $F$-region $\omega_{8}=\omega_{n}$.

In the middle-latitude $E$-region of ionosphere they appear in the form of well observed large-scale perturbations MLO (middle-latitude long-period oscillations) and in the $F$-region in the form of MIWP (magnetic-ionospheric wave perturbations). The variety of different features of magnetogradient planetary waves considered above opens a prospect for the more detailed investigation of the large-scale perturbations of electromagnetic nature regularly observed in the $E$ and $F$-regions of ionosphere both in the calm (background oscillations) and in the perturbed ionosphere (forced oscillations).
Thus it is possible to consider that all eigenfrequencies of ionospheric resonator in the MHD approximation (system of the eighth time order) are completely covered. At the same time the eighth eigenfrequency, as follows from the above is present in all three components of ionospheric medium and like other known wave modes it is the direct consequence of the equations of MHD of the ionosphere.

This work has been partially supported by the Russian Foundation for Basic Research (Project \# 10-02-00875).

\section{REFERENCES}

[1] Pedloski J. Geophysical fluids dynamics. Springer-Verlag: New York, Heidelberg Berlin 1982.

[2] Landau LD, Lifshits EM. Hydrodynamics. Nauka, Moscow, 1988 (translated in English).

[3] Khantadze AG. Some questions of the dynamics of the conducting atmosphere. Tbilisi. Science 1973.

[4] Rossby CG. On the nature of the general circulation of the lower atmosphere. In: The atmosphere of the earth and planets. Ed, Kuiper GP. Univ. of Chicago Press 1949.

[5] Gill A. Atmosphere-ocean dynamics. Academic Press, New York 1986.

[6] Khantadze AG. Determination of the wind field by the pressure gradient field and latitudinal effect of geomagnetic field. Proc Inst Geophys Acad Sci Georgian SSR 1967: 24-9 (in Russian).

[7] Khantadze AG, Jandieri GV, Ishimaru A, Kaladze TD, Diasamidze ZhM. Electromagnetic oscillations of the Earth's upper atmosphere (review). Ann Geophys 2010; 28: 1387-99.

[8] Tolstoy I. Hydromagnetic gradient waves in the Ionosphere. J Geophys Res 1967; 47 (5): 1435-42.

[9] Burmaka VP, Kostrov LS, Chernogor LF. Statistical characteristics of Doppler HF radar at sounding of the middle ionosphere perturbed by launching of spacecrafts and solar terminator. Radiophys Radio Astron 2003; 8(2): 143-62.

[10] Khantadze AG, Aburjania GD, Gvelesiani AI. Physics of generation of the new branches of planetary electromagnetic waves in the ionosphere. Geomagnet Aeron 2003; 43(2): 193-203.

[11] Aburjania GD, Jandieri GV, Khantadze AG. Self-organization of planetary electromagnetic waves in the E-region of the ionosphere. J Atmos Solar-Terrastrial Phys 2003; 65: 661-74.

[12] Khantadze AG, Aburjania GD, Jandieri GV. On the mechanism of the vortical electric field generation in the E-region of ionosphere. Plasma Physics Report 2004; 30(1): 81-90.

[13] Khantadze AG, Jandieri GV. General-planetary character of threedimensional planetary waves propagation in lower and upper Earth atmosphere. J Atmos Solar-Terrastrial Phys 2009; 71: 45-8.

[14] Aburjania GD, Chargazia KZ, Jandieri GV, et al. On the new models of planetary scale electromagnetic waves in the ionosphere. Ann Geophys 2004; 22(4): 1203-11.

[15] Yaglom AM. Dynamics of the large-scale processes in the barotropic atmosphere. Proc Acad Sci USSR, Izv. Acad Nauk Geol 1953; 4: 347-69.

[16] Monin AS, Obukhov AM. Small oscillations of atmosphere and adaptation of meteorological fields. Proc Acad Sci USSR, Izv. Acad. Nauk Geol 1958; 11: 1360-73.

[17] Cavalieri DJ, Deland RJ, Poterna JF, Gavin RF. The correlation of VLF propagation variations with atmospheric planetary-scale waves. J Atmos Terr Phys 1974; 36: 561-74.

[18] Sharadze ZS. Phenomena in the middle-latitude ionosphere. PhD Thesis. Moscow, Russia 1991.

[19] Fagundes PR, Pillat VG, Bolzan MJA. Observations of F layer electron density profiles modulated by planetary wave type oscillations in the equatorial ionospheric anomaly region. J Geophys Res 2005; 110: 1302-12.

[20] Kazimirovskii ES, Kokourov VD. Motions in the ionosphere. Nauka: Novosibirsk, Russia 1979 (in Russian).

[21] Al'perovich LS, Ponomarev EA, Fedorovich GV, Geophysical phenomena modeling by an explosion: a review, Izv. Phys Solid Earth 1985.

[22] Jacchia LG. Thermospheric temperature, density and composition: new models. Spec Rep Smithsonian Astrophys Obser 1977; 375: 1106. 
[23] Gershman BN. Dynamics of the ionospheric plasma. Nauka: Moscow, Russia 1974 (in Russian).

[24] Akasofu SI, Chapman S. Solar-Terrestrial Physics, Oxford Univ. Press. Oxford 1972.

[25] Hajkowicz LA, Global onset and propagation of large-scale traveling ionospheric disturbanced as a result of the great storm of 13 March 1989. Planet Space Sci 1991; 39: 583-93.
[26] Drobzhev VI, Molostov GF, Rudina MP, et al. Ionospheric response on perturbations activation by industrial explosions. Ionospheric Investig 1986; 33: 61-71 (in Russian).

[27] Kadomtsev BB. Collective Phenomena in Plasma. Science, Moscow 1976 (in Russian).

[28] Cowling TG. Magnitohydrodynamics, New York, London 1957.

[29] Holton JR. The dynamic meteorology of the stratosphere and mesosphere. Am Meteor Soc. Boston, Massachusetts 1975.

(C) Jandieri et al.; Licensee Bentham Open.

This is an open access article licensed under the terms of the Creative Commons Attribution Non-Commercial License (http: //creativecommons.org/licenses/by$\mathrm{nc} / 3.0 /$ ) which permits unrestricted, non-commercial use, distribution and reproduction in any medium, provided the work is properly cited. 